\title{
Contemporary narrative review on left atrial strain mechanics in echocardiography: cardiomyopathy, valvular heart disease and beyond
}

\author{
Vardhmaan Jain $^{1}$, Raktim Ghosh ${ }^{2}$, Manasvi Gupta ${ }^{3}$, Yoshihito Saijo ${ }^{4}$, Agam Bansal ${ }^{1}$, Medhat Farwati ${ }^{1}$, \\ Rachel Marcus ${ }^{2}$, Allan Klein ${ }^{4}$, Bo Xü ${ }^{4}$ \\ ${ }^{1}$ Department of Internal Medicine, Cleveland Clinic, Cleveland, OH, USA; ${ }^{2}$ MedStar Heart and Vascular Institute at MedStar Union Memorial \\ Hospital and MedStar Good Samaritan Hospital, Baltimore, MD, USA; ${ }^{3}$ Department of Internal Medicine, UConn Health, University of \\ Connecticut, Farmington, CT, USA; ${ }^{4}$ Section of Cardiovascular Imaging, Robert and Suzanne Tomsich, Department of Cardiovascular Medicine, \\ Sydell and Arnold Miller Family Heart, Vascular and Thoracic Institute, Cleveland Clinic Foundation, Cleveland, OH, USA \\ Contributions: (I) Conception and design: V Jain, B Xu, R Ghosh; (II) Administrative support: None; (III) Provision of study material or patients: \\ None; (IV) Collection and assembly of data: V Jain, M Gupta, R Ghosh, Y Saijo, A Bansal, M Farwati, B Xu; (V) Data analysis and interpretation: V \\ Jain, M Gupta, Y Saijo, A Bansal, A Klein, B Xu; (VI) Manuscript writing: All authors; (VII) Final approval of manuscript: All authors. \\ Correspondence to: Bo Xu, MD, FRACP, FACC, FASE. Section of Cardiovascular Imaging, Robert and Suzanne Tomsich, Department of \\ Cardiovascular Medicine, Sydell and Arnold Miller Family Heart, Vascular and Thoracic Institute, Cleveland Clinic, 9500 Euclid Avenue, Desk J1-5, \\ Cleveland, OH 44195, USA. Email: xub@ccf.org.
}

\begin{abstract}
Left atrial (LA) strain mechanics refer to the measurement of LA myocardial deformation expressed as a percentage, and have been gathering interest over the last decade with expanding research supporting their utility in multiple cardiovascular disorders. Measured through advanced dynamic imaging techniques which include tissue Doppler imaging (TDI) and two-dimensional (2D) speckle tracking echocardiography (STE), LA strain mechanics are affected by left ventricular diastolic dysfunction prior to the onset of functional and structural changes in the left ventricle (LV). There is a need for practising cardiologists to become more familiar with the clinical utility of LA strain mechanics. In this article, we begin by reviewing the physiologic function of the LA, using this as a basis for understanding LA strain mechanics. The focus of this review article is to provide a contemporary update on the utility of LA strain mechanics in a range of cardiovascular disorders, including atrial fibrillation (AF), hypertrophic cardiomyopathy (HCM), valvular pathologies, coronary artery disease (CAD) as well as systemic diseases, such as hypertension (HTN), obesity and diabetes mellitus (DM). This article also highlights the current limitations in more widespread clinical applications of LA strain mechanics, as well as outlining the future perspectives on the clinical applications of LA strain mechanics.
\end{abstract}

Keywords: Left atrium (LA); left atrial mechanical function (LA mechanical function); strain imaging; strain rate imaging; echocardiography

Submitted Apr 29, 2020. Accepted for publication Aug 19, 2020.

doi: $10.21037 / \mathrm{cdt}-20-461$

View this article at: http://dx.doi.org/10.21037/cdt-20-461

\section{Introduction}

The left atrium (LA) plays an essential role in left ventricular filling. Evaluation of left atrial (LA) structure and function has an important place in diagnosis and prognostication of a variety of disease processes, such as atrial fibrillation
(AF), hypertension (HTN), heart failure (HF), valvular heart disease, cardiomyopathies and coronary artery disease (CAD). The assessment of LA function in physiologic and pathologic states is increasingly being viewed as a biomarker for outcomes in various cardiovascular conditions (1). 


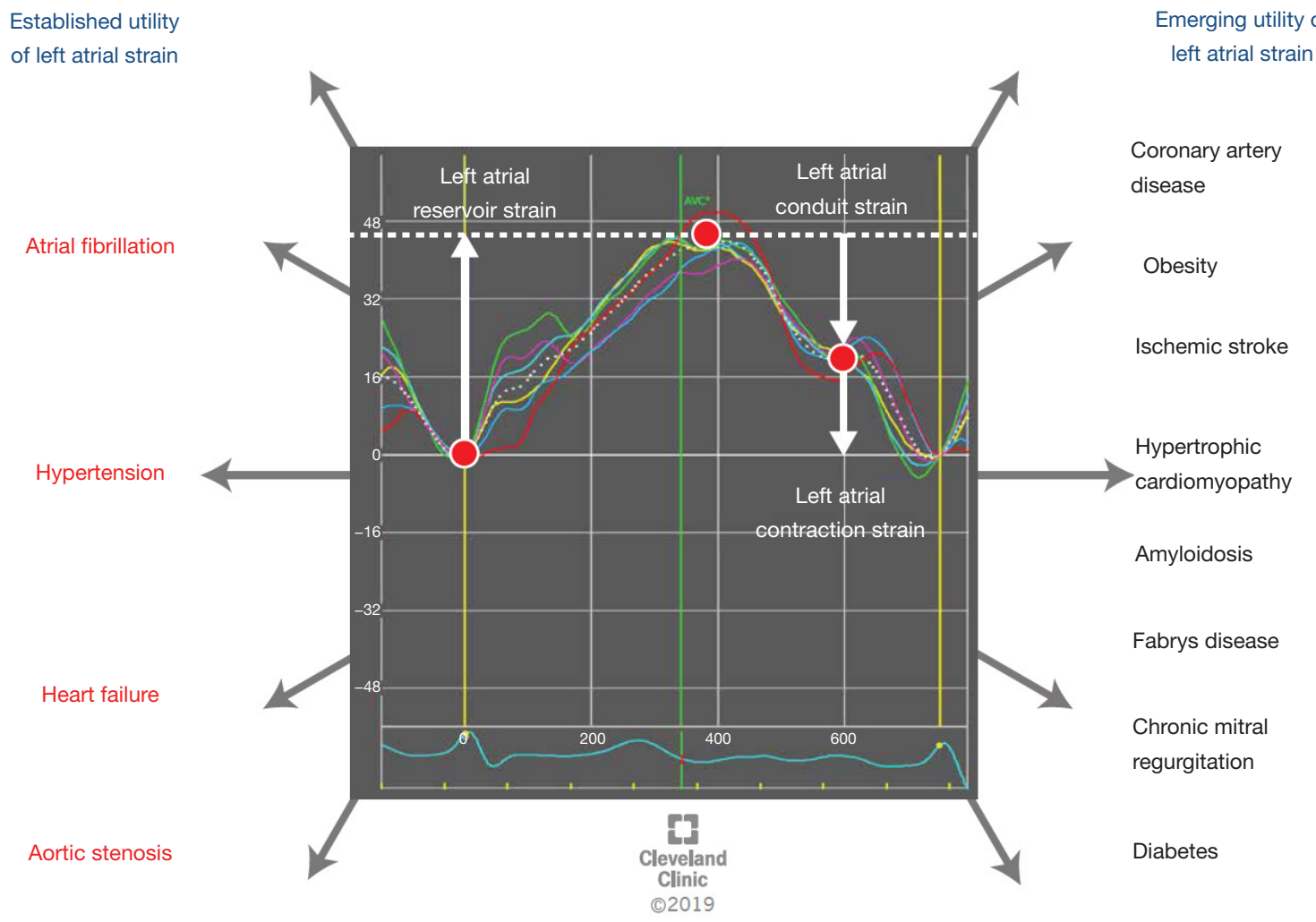

Figure 1 Central illustration demonstrating the established and emerging applications of LA strain mechanics in various cardiovascular conditions. LA, left atrial.

Myocardial strain is a measure of tissue deformation, and is expressed as a percentage change in the length of the underlying myocardial tissue. Strain imaging, using twodimensional (2D) speckle tracking echocardiography (STE), has emerged as a non-invasive and reliable marker of estimating myocardial function, and helps overcome a number of angle-dependent limitations of traditional Doppler echocardiography (2). Strain imaging using tissue Doppler imaging (TDI) as well as 2D STE allows an early estimation of myocardial dysfunction, viability, evaluating optimal timing of cardiac surgery, as well as post-surgical outcomes (3). In recent times, strain and strain rate imaging have been developed as non-invasive techniques to assess systolic and diastolic function of the LA. Here, we focus on the various aspects of strain imaging, how disease processes affect LA strain parameters, and discuss the various clinical applications of LA strain mechanics (Figure 1). We present the following article in accordance with the NARRATIVE REVIEW reporting checklist (available at http://dx.doi. org/10.21037/cdt-20-461).

\section{Physiological function of the LA in cardiac cycle and correlation with left ventricular function}

The physiologic function of the LA in assisting left ventricular filling, and subsequently cardiac output, can broadly be studied under three phases: the LA "reservoir" phase, the passive "conduit" phase, and the "contractile" phase (4). Initially, during the iso-volumetric relaxation phase, the LA relaxes and receives blood from the pulmonary veins, hence acting as a "reservoir" of potential energy. In this sense, the LA "reservoir" strain is a reflection of LA behavior during the period when the mitral valve is closed, and may be sensitive to changes in LA compliance as seen with fibrosis. Following the opening of the atrioventricular valves in early ventricular diastole, the LA acts as a "conduit" and allows transfer of blood from the pulmonary veins to the left ventricle (LV). Thus, the LA "conduit" strain is a reflection of LA behavior when the mitral valve is opened, and may be affected by changes in the $\mathrm{LV}$, as seen in $\mathrm{HF}$ and long-standing hypertensive heart 
disease. Finally, in late ventricular diastole that coincides with the atrial contraction phase, the LA acts as a "pump" to augment left ventricular filling by 20-25\% (5). LA strain measured during this phase is a measure of the contractile function of the LA and may be affected by changes in both LA and LV. Through these three phases, the LA plays an essential role in the continuous transfer of blood from the pulmonary veins to the $\mathrm{LV}$ in different phases of the cardiac cycle.

The myocardial deformation or strain in the LA is load dependent and varies with LV function. Physiologically, LA strain changes are often noted prior to the onset of functional or structural changes in the LV. The changes in myocardial deformation detected are described under each section, and are often secondary to pressure changes in LV.

\section{Existing imaging techniques for evaluating the LA}

The contributions of the LA reservoir, conduit and pump functions to left ventricular filling are approximately $50 \%$, $30 \%$ and $20 \%$, respectively, in healthy individuals (6). Alteration in LA size and function can occur in a number of physiological as well as pathological disease processes. It is, therefore, important to have non-invasive and efficient methods for estimation of LA function. M-mode echocardiography can measure the LA diameter in the antero-posterior diameter; however, it cannot be extrapolated to estimate the LA size accurately due to the irregular shape of the chamber $(7,8)$. Measurement of LA volume by 2D and three-dimensional (3D) echocardiography provides a more accurate estimate, comparable to reference standards such as multi-detector cardiac computed tomography (MDCT) and cardiac magnetic resonance (CMR) imaging (9-11). The pitfalls of $3 \mathrm{D}$ echocardiography potentially include inconsistent estimation of LA border and LA long axis, wrongly timed reading frame, falsely foreshortened LA, and inter-observer and intra-observer disparities (1).

Pulsed wave Doppler also provides a valuable measure of transmitral and transpulmonary venous blood flow, as well as LV filling pressures which help determine LA function $(12,13)$. The peak atrio-ventricular flow A-wave velocity, its velocity-time integral, atrial ejection force, and atrial ejection fraction are some of the parameters applied to assess LA function indirectly $(14,15)$. However, the majority of the listed parameters require the presence of normal sinus rhythm, precluding their use in AF and other abnormal rhythms leading to the development of the rhythm independent variable called LA functional index (LAFI). LAFI is defined as LAFI = LAEF $\times$ LVOT VTI/ LAESVI, where LAEF is LA emptying fraction, LVOT VTI is velocity time integral across the left ventricular outflow tract and LAESVI is the largest LA volume measured in ventricular systole indexed to body surface area. Thus, it is proportional to the LA reservoir function and stroke volume, and inversely proportional to LA size (16). LAFI is found to be lower in patients with AF and improves on cardioversion to sinus rhythm (16). A recent study investigating its utility to existing prediction scores for cardiovascular disease (CVD) did not yield positive results (17).

\section{LA strain imaging}

Strain imaging has traditionally been studied for assessing LV function. Recently, strain imaging has been applied to assessing LA function. It allows for measurement of LA function through the different phases of the cardiac cycles, and differentiates between active and passive myocardial involvement. While utilizing strain measurement to assess LA function, it is also important to consider the impact of LA size and wall thickness in accordance to LaPlace's law as an independent variable. Pre-clinical studies have shown that because of the heterogeneous thickness and complex anatomy of the LA, its effect on strain is difficult to assess, but in general, this effect is more pronounced in the LA passive filling phase than in the contractile phase, and more near the annulus than the lateral walls $(18,19)$. Further, there may be heterogeneity in the value of LA strain measured by different software packages, as described later. Prospective studies addressing these studies may help strengthen the role of LA strain measurement in clinical practice.

LA strain can be measured using two main techniques: TDI, or 2D STE. Image acquisition should be at a higher frame rate when using TDI, ideally $>140$ frames per second compared to STE where the frame rate is often between 40-80 frames per second. At least four QRS complexes should be covered with data sampling starting at least $100 \mathrm{msec}$ before the $\mathrm{R}$ wave of the first QRS complex and ending about $200 \mathrm{msec}$ after the last QRS complex. In the absence of existence of dedicated software for LA strain analysis, LV strain software are used to analyze the LA mechanics after image acquisition. These include vendor specific software such as EchoPAC (GE Healthcare, Chicago, IL, USA) 
and vendor independent software such as Velocity Vector Imaging (Siemens Healthineers, Washington, DC, USA). Notably, there are no American Society Echocardiography (ASE) consensus guidelines at present regarding acceptable variability across different software platforms for LA strain analysis (20). Wang et al. examined the variability across vendor platforms in LA measurement and concluded that vendor independent software had a significantly smaller difference in strain measurements (21).

The European Association of Cardiovascular Imaging/ American Society of Echocardiography hare commend recommended LA strain analysis should begin with tracing the endocardial border of the mitral annulus, and then tracing the LA endocardial border, extrapolating across the pulmonary veins and/or LA appendage (LAA) orifices, up to the opposite side of the mitral annulus, using the apical four chamber view (22). Biplane LA strain (from four and two chamber views) could also be considered as an option (22). The myocardial region of interest of the LA is defined by the endocardial border and the epicardial border, similar to $\mathrm{LV}$ strain measurement (22). If the tracking software requires the definition of the region of interest, a default width of $3 \mathrm{~mm}$ is recommended (22). The region of interest size and shape should be adjusted in order to include the thickness of LA wall and to avoid including the pericardium (22). If the tracking software uses endocardial tracking only, the endocardial contour is delineated (22). The user should compare visually the tracking to the motion of the underlying LA wall in order to judge the accuracy of the LA strain estimate (22). Feasibility of LA strain measurements was reported in a meta-analysis, ranging from $83.3 \%$ to $96.7 \%$ on a per-patient bias, and from $93.8 \%$ to $98.1 \%$ on a per-segment bias (23).

As the LA cycles through different phases, the strain measurements change accordingly. Strain measured during the LA filling phase corresponds to reservoir strain (LASr) (19). This is followed by a fall in the strain curve and passive flow of blood from the LA to LV, during the conduit phase of the LA. Strain measured in this phase corresponds to the conduit strain (LAScd). Finally, there is a second rise on strain curve with contraction of the LA during the booster phase and peak LA strain measured in this phase is known as LA contractile strain (LASct).

A recent study evaluated the pattern of change of tissue velocity as well as the strain and strain rate changes across the different walls of LA amongst healthy volunteers. They reported that the peak systolic and absolute diastolic velocities decreased from the base to mid-wall and from the mid-wall to the roof, and the value of strain and strain rate increased from the base to mid-wall and from the mid-wall to the roof (24). Many studies have attempted to suggest standard values for LA strain, and the largest involving 329 participants from 10 centers showed that mean value for LA peak systolic strain during reservoir phase was $45.5 \% \pm 11.4 \%$, and strain rate during peak atrial contraction was $-2.11 \pm 0.61 \mathrm{~s}^{-1}(25)$.

\section{Clinical applications of LA strain mechanics}

\section{LA strain and $A F$}

AF is one of the most commonly encountered electrophysiological abnormalities and is associated with increased cardiovascular morbidity and mortality. A high $\mathrm{CHA}_{2} \mathrm{DS}_{2}$-VASc score coupled with transesophageal echocardiographic (TEE) findings of LA or LAA thrombus or spontaneous echo contrast (SEC) have been shown to have a positive correlation with increased thrombo-embolic risk in patients with $\mathrm{AF}$ (26). Large prospective studies have shown a positive correlation between increasing LA dimensions and volume, as measured on M-mode and 2D echocardiography, and the risk of developing AF $(27,28)$. These results encourage further exploration into the utility and accuracy of estimating LA strain through noninvasive methods.

A recent study in patients undergoing ablation for persistent AF showed that decreasing LAA emptying velocity and worsening LASr were associated with findings of LAA thrombus on TEE (29). In patients with advanced interatrial block, worsening LA strain rate in the systolic pump function has been shown to be associated with newonset $\mathrm{AF}$ and ischemic stroke (30). Worsening LA systolic strain has also been associated with progression from paroxysmal to persistent AF (31).

Following catheter ablation pulmonary vein isolation, there remains a risk of AF recurrence. Studies correlating LA strain in patients undergoing catheter ablation for AF have shown that lower systolic strain rates may be associated with recurrence of $\mathrm{AF}$ after catheter ablation, and pre-procedural screening using strain analysis may have value in selection of patients suitable for the procedure. In an age and gender matched cohort of 40 patient with $\mathrm{AF}$ undergoing catheter ablation, Hwang et al. showed that LA strain was useful for predicting AF recurrence at 9 months follow-up (32). Such results point toward the potential utility of LA strain measurement in prognosticating AF recurrence after catheter ablation. 


\section{LA strain in HTN}

HTN results in left ventricular pressure overload and subsequent changes in LA structural and morphological properties (33). Structural changes in the LA referred to as remodeling, are often preceded by functional changes as measured by strain and strain rate. In a study including patients with diabetes mellitus (DM) and HTN who have normal LA volume measurements, Mondillo et al. showed that LA deformational parameters, including LASr, as well as diastolic strain was reduced in patients with DM and HTN when compared to controls (34). Further, the presence of both clinical conditions resulted in additive decline in the LA strain parameters (34). Similar findings were confirmed by Hennawy et al., who showed that the parameters of LA function, including LASr and LA deformation index were significantly lower in hypertensive patients with normal LA volume indices as compared to controls. Their study was unique in the way that the control subjects were matched for a greater number of confounding factors, including body mass index (BMI), which was not accounted for in prior studies (35). Additional studies in individuals with preserved $\mathrm{LV}$ function have confirmed these findings, giving merit to the role of LA strain measurement in detecting HTN related myocardial dysfunction, before the onset of LV wall thickening (36). Pharmacological interventions resulting in improvement in LA strain parameters with better blood pressure control has also been demonstrated, pointing toward a beneficial role of LA strain measurement to monitor response to treatment (37).

\section{LA strain in $\mathrm{HF}$}

With growing interest in HF, there has been expanding interest in diagnostic and prognostic implications of various imaging modalities. Doppler imaging is angle-dependent and has a debatable value in estimating left ventricular filling pressure changes (38). As compared to E/e' and $\mathrm{E} / \mathrm{Em}$, the global LASr has greater accuracy in estimating left ventricular end diastolic pressure (LVEDP), particularly pulmonary capillary wedge pressures (39-41). In a study evaluating LA reservoir function in 405 patients with $\mathrm{HF}$ with reduced ejection fraction (HFrEF), impaired LASr was found to be associated with elevated left ventricular and atrial volumes, worse left ventricular global longitudinal strain and LVEF, worse right ventricular systolic dysfunction and more severe diastolic dysfunction. These changes in echocardiographic variables translated into LASr being a significant predictor of death/HF hospitalization at 12, 24 and 36 months of follow-up (42). Other studies have also demonstrated the association of impaired LASr with cardiovascular mortality, risk of AF, higher brain-derived natriuretic peptide (BNP) levels and worsening New York Heart Association (NYHA) in patients with HFrEF (42-44).

LA strain imaging has also shown promise in HF with preserved ejection fraction (HFpEF), with some differences from $\mathrm{HFrEF}$ by virtue of its distinctive physiology.

Impaired left ventricular distensibility causes a compensatory and chronic elevation in LA pressure, eventually leading to declining reservoir and pump functions along with increased LA stiffness $(45,46)$. In a prospective cohort study, Telles et al. suggested that impaired LA strain could be used as a marker of impaired exercise hemodynamics (47). Patients diagnosed with HFpEF by right heart catheterization have reduced LASr, increased stiffness, worse pump strain and a reduced contraction strain index (47). Impaired LA reservoir and pump phase function are also strongly associated with increased pulmonary artery pressure and resistance, and reduced cardiac output and exercise workload. However, the usefulness of LA strain in predicting hospitalization and death, independent of LV strain, remains uncertain $(48,49)$.

\section{$L A$ strain and aortic stenosis $(A S)$}

LA enlargement is a common consequence of AS and estimation of LA size is an independent determinant of left ventricular filling pressure (12). Although data from recently published small scale trials has also indicated a correlation between these indices, such as LA volume index (LAVI), LA distensibility, LA strain and LA volume, and the chronicity of the disease, the value of LA strain in prognostication of AS remains unclear. In the absence of primary aortic or mitral disease, LA diameter has been positively correlated with post valve replacement outcomes in AS (50). Rusinaru et al., in a recent French study, studied 715 patients with aortic leaflet calcification and a peak aortic jet velocity of $>2.5 \mathrm{~m} / \mathrm{s}$ for a median period of 22 months to estimate the cut-off value of LAVI, which can be used to correlate with the risk for the combined endpoint of cardiovascular death and hospitalization for HF. The results of the study indicated that LAVI of $\geq 45 \mathrm{~mL} / \mathrm{m}^{2}$ was associated with increased left ventricular volume and filling pressure (51). A subsequent study evaluated 77 severe AS and 25 moderate AS patients for a median follow-up period of 25 months, demonstrating that LA distention of $\leq 69 \%$ and LA 
maximal strain of $\leq 17 \%$ were independently associated with increased hospitalization for HF and all-cause mortality (52).

\section{$L A$ strain and CAD}

As with other CVD processes, ischemic effects on myocardium also leads to deformation of LA myocardium before the onset of gross structural or functional changes (53). In one of the earliest studies on CAD patients, Yan et al. showed that LA strain/strain rate was affected in patients, even in those with preserved ejection fraction and normal LA size. LASr was decreased in patients with CAD with a significant increase in atrial strain in late $\mathrm{LV}$ filling, particularly in patients with left anterior descending (LAD) stenosis (54). In a small-scale study conducted in Egypt based on 30 patients with stable $\mathrm{CAD}$, it was found that $\mathrm{LA}$ diameter and volume correlated directly with the severity of CAD. Additionally, LA strain and strain rate were noted to be reduced in CAD and negatively correlated with increasing severity of stenotic lesion as measured by Syntax score (55). Existing literature also indicates a lower early diastolic LA strain rate and higher late diastolic strain rate in diabetic $\mathrm{CAD}$ as compared with non-diabetics $\mathrm{CAD}$ (56). Although the existing data are largely from small scale studies, it opens up possibilities for future exploration of LA indices in CAD and early evaluation for both medical and interventional therapies.

\section{LA strain and obesity}

Obesity is associated with early LA enlargement partly as a compensatory response to increased cardiac output and stroke volume, similar to what may be observed in athletes $(57,58)$. Other mechanisms contributing to atrial remodeling include increased afterload, myopathic effect of systemic inflammation and adipokines, and a possible paracrine effect from epicardial fat $(59,60)$. Therefore, using LA dimensions as a marker of obesity related LA remodeling offers little insight into the causal mechanism. In a community-based design, the Chirinos et al. designed a first of its kind study to assess the change in LA strain patterns with increasing BMI amongst 1,531 participants (59). Authors of the study reported that increasing BMI was associated with declining LA reservoir and conduit function, and a compensatory increase in the booster pump function, when adjusted for age and gender (59). They further argued that the loss of the booster pump function with increasing BMI likely increased likelihood of development of HF (61). LA strain parameters can, thus, be used to trend obesity related cardiac remodeling, and monitor response to medical and lifestyle interventions to decrease predisposition for adverse outcomes.

\section{LA strain in acute ischemic stroke}

Stroke is a leading cause of long-term cardiovascular morbidity and mortality, as well as healthcare expenditure in the western world. In patients with $\mathrm{AF}$, the $\mathrm{CHA}_{2} \mathrm{DS}_{2}-$ VASc score is used for risk stratification and assessment of the need for anticoagulation (62). The score does not take into account the role of LA size and deformational parameters in determining the risk of stroke in patients with AF. Studies evaluating LA strain in patients with paroxysmal and persistent $\mathrm{AF}$ have shown that reduced strain values are associated with higher atheroembolic burden, and provided incremental value over $\mathrm{CHA}_{2} \mathrm{DS}_{2}$-VASc score in risk stratification (63).

Moreover, studies in patients with acute ischemic stroke without a prior diagnosis of $\mathrm{AF}$ have shown that reduced global LA peak longitudinal strain offered prognostication value in terms of recurrent stroke, and was suggestive of more advanced atrial cardiomyopathy (64). These patients were also shown to be more likely to develop subsequent $\mathrm{AF}$, suggesting that altered LA contractility predisposed to increased risk of developing $\mathrm{AF}$ as well as thromboembolic phenomenon (65). In theory, the reduced value of global PLAS could strengthen the indication for loop recorder implantation (to detect possible atrial arrhythmias) and/ or for anticoagulation therapy for secondary prevention in patients with a recent acute ischemic stroke.

\section{LA strain in hypertrophic cardiomyopathy (HCM)}

HCM is one of the most common inherited cardiomyopathies characterized by heterogeneous myocardial hypertrophy and left ventricular dysfunction (66). In a manner reminiscent of changes in AS, the natural course of the disease involves progressive LV diastolic dysfunction, leading to increased filling pressures which are transmitted to the LA and cascading to progressive LA enlargement with morpho-functional dysfunction (67). The manifestations of the disease encompass decreased exercise tolerance, $\mathrm{HF}, \mathrm{AF}$, ventricular arrhythmias, and sudden cardiac death (68-70). It is therefore clinically relevant to risk stratify these patients using non-invasive imaging modalities. 
There have been a number of studies demonstrating that increasing left ventricular stiffness and subsequent decrease in LV long-axis shortening result in worsening LV longitudinal strain values, often associated with adverse outcomes (71). Early reports have also shown that the disease process is associated with progressive LA stiffness and decreased reservoir function forming the basis for the growing interest in evaluating morpho-functional changes in LA in patients with HCM (72). Figure 2 demonstrates representative LA strain curves analyzed by two different software platforms (EchoPAC and Velocity Vector Imaging) in a healthy subject versus a patient with HCM. In HCM, the LA strain values were lower, compared with the normal subject. In a study of $76 \mathrm{HCM}$ patients, Fujimoto et al. showed that HCM was associated with declining LA booster as well as conduit strain parameters, and increasing LAVI. Worsening of LA pump function was associated with increasing hospitalizations for $\mathrm{HF}$ and $\mathrm{AF}$, and correlated with adverse outcomes (73). Another study assessing LA dysfunction among different phenotypes of HCM found that LA function was impaired even in minimally symptomatic and non-obstructive phenotypes, and progressively worsened with the degree of obstruction (74). These findings support the utilization of LA strain assessment as a means of tracking disease progression and risk stratification of patients, with the hope of improved survival with timely interventions. However, it is important to note that Wang et al. recently showed that there may be variability in LA strain measurements by different strain software platforms (21). Figure 2 demonstrates an example of variability in strain mechanics between EchoPAC and Velocity Vector Imaging. Therefore, when interpreting LA strain values, attention must be paid to the type of software platform used (75).

\section{$L A$ strain in amyloidosis (AL)}

Cardiac amyloidosis (CA) involves the intramyocardial deposition of abnormally folded amyloid chains, and is usually a component of systemic AL (76). Based on the type of amyloid chain deposited, CA can be light chain AL, where clonal expansion of plasma cells leads to overproduction of immunoglobulin light chain, or transthyretin AL, which can be further due to hereditary (ATTRm) or deposition of wild type (ATTRwt) transthyretin (77). Regardless of the etiology, CA results in progressive increases in LV thickness, diastolic dysfunction, and elevated filling pressures (78). This increased pressure coupled with deposition of amyloid fibrils leads to progressive LA dilation and dysfunction, and worsening LA size has been correlated with adverse outcomes in patients with CA (79).

A control matched study of 77 patients with diagnosed AL type of CA using 3D speckle tracking imaging (STI) showed that advancing disease was associated with lower 3D-LA total emptying fraction (3D-tLAEF), and worse LASr. The 2-year survival was noted to be significantly lower in patients with $3 \mathrm{D}$-tLAEF $<+34 \%(\mathrm{P}=0.003)$ and in those with $\mathrm{LASr}<+14 \%(\mathrm{P}=0.034)(80)$. Another study by Nochioka et al. found that all LA strain parameters, including peak longitudinal strain rate (LSR) (reservoir function), early LSR (conduit function), and late LSR (booster pump function) were impaired in individuals with CA regardless of the characteristic of fibrils deposited (AL, ATTRm, ATTRwt). LA dysfunction correlated well with the degree of $L V$ dysfunction, and was worse in patients with ATTRwt, compared with other subtypes of CA (81).

\section{$L A$ strain in Fabry disease (FD)}

FD is an X-linked disorder characterized by decreased activity of the lysosomal enzyme alpha-galactosidase, resulting in deposition of glycosphingolipids in multiple organ systems, and cardiovascular involvement is a cause of significant morbidity and mortality, including premature death (82). Enzyme replacement therapy (ERT) has been shown to improve mortality and reverse increased $L V$ wall thickness. However, due to the high cost, it is currently recommended only in patients with increased $L V$ wall thickness, diastolic dysfunction and LA enlargement (83). A study by Pichette et al. enrolling 50 control matched subjects with FD noted that LASr, early diastolic strain and late contractile strain were significantly impaired in patients with FD (84). Fifteen patients who received ERT had significant improvement in all LA strain parameters when compared to 15 patients who did not receive ERT (82). The peak positive and early diastolic strain were also found to correlate with adverse outcomes like new onset $\mathrm{AF}$ and stroke at 4-year follow-up (84). Such findings highlight the important role of LA strain imaging in early assessment of disease severity and timely initiation of ERT for better outcomes. It is noteworthy that a number of conditions including HTN, HCM, CA and FD may lead to LV thickening and consequently affect LA strain patterns, but at present data are limited regarding the feasibility of using the pattern of LA strain mechanics to determine the cause of $\mathrm{LV}$ thickening. 
Echo PAC

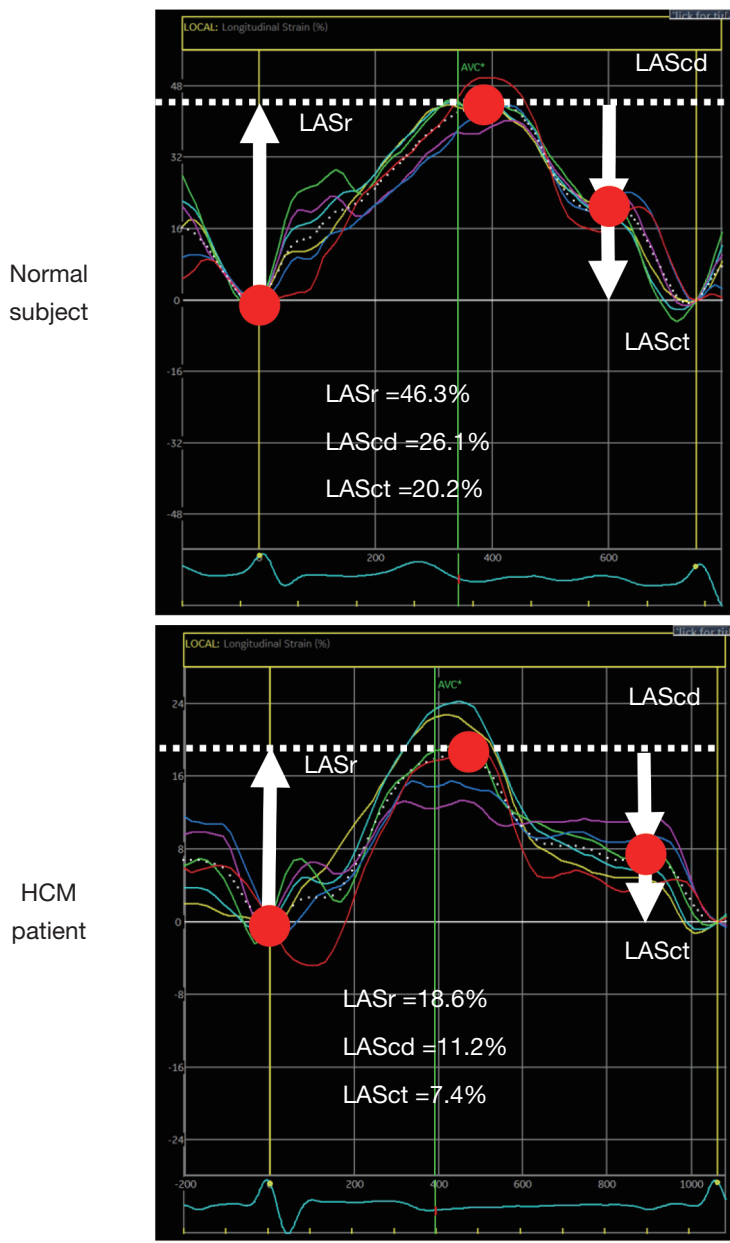

Velocity vector imaging
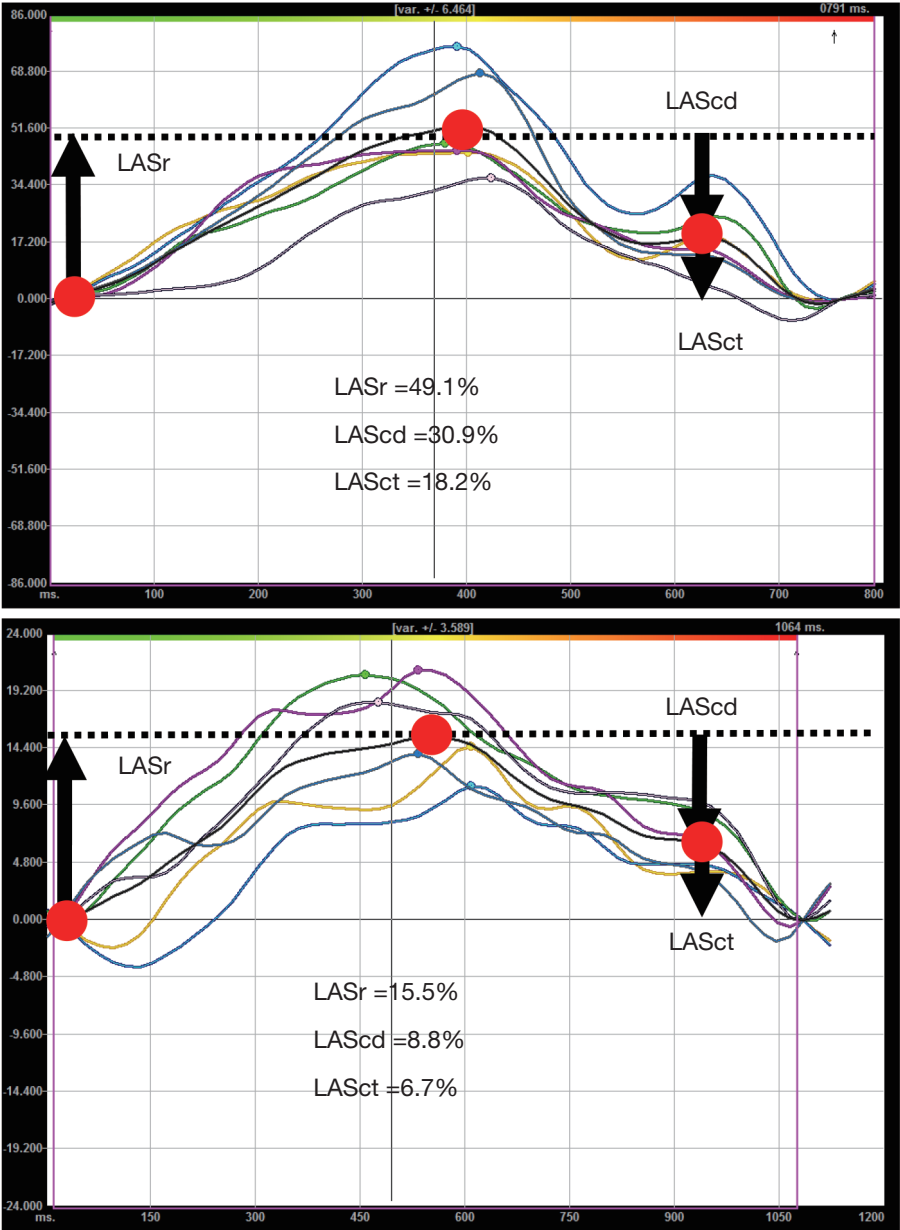

Figure 2 LA strain mechanics through the different phases of the cardiac cycle in a normal subject (top panel) compared to a patient with HCM (bottom panel). The series of images on the left represent strain measurements by EchoPAC (GE Healthcare, Chicago, IL, USA)] software, while the series of images on the right represents measurements by velocity vector imaging (Siemens Healthineers, Washington, DC, USA). The illustration also highlights the differences in LA strain values obtained, depending on the type of software platform used. LA, left atrial; HCM, hypertrophic cardiomyopathy; LASr, LA reservoir strain; LAScd, LA conduit strain; LASct, LA contractile strain.

\section{$L A$ strain and chronic mitral regurgitation (MR)}

LA dilation seen in chronic MR is associated with increased risk of development of new onset $\mathrm{AF}$, which is also a class IIb recommendation for mitral valve replacement (85). Yang et al. concluded that reduced baseline LA strain and strain rate in the filling phase were associated with a higher rate of mortality and mitral valve replacement or repair procedures after a mean follow-up of 13.2 months in primary severe MR (86). A small study on participants with severe Carpentier class II MR with lower baseline LASr rate were noted to have more rapid LA remodeling over a mean period of 153 months (87). In a study conducted on 192 patients with mitral valve prolapse and MR, it was noted that tLAEF, reservoir strain and the contractile strain were all independent predictors of requirement of valve replacement. In fact, the study calculated a sensitivity of $91 \%$ and a specificity of $92 \%$ for future surgery with tLAEF cutoff value of $<50 \%$ (88). These findings, although restricted to small studies, reflect a growing utility of LA strain measurements in stratifying risk, predicting progression and eventually optimizing timing of surgical interventions in this patient population. 


\section{$L A$ strain and DM}

DM is a common cardiovascular risk factor, often found in association with HTN, with current data indicating worldwide prevalence of 7\% by 2030 (89). Long standing DM is associated with LA enlargement in concordance with LV diastolic dysfunction and an increased predisposition to HF (90). Studies using LA volume measures and strain echocardiography have shown decreased LA reservoir and conduit function, in addition to impaired global longitudinal LV strain (91). In a recent study evaluating 73 patients with type $2 \mathrm{DM}$, Kadappu et al. showed that LA enlargement was independent of the degree of HTN and diastolic dysfunction (92). Another study highlighted the usefulness of LA strain mechanics in detecting early changes in the myocardium caused by DM, reporting impaired LA strain mechanics (reservoir and conduit strains) in diabetic patients without HTN and LV hypertrophy (93). Tadic et al. performed a study in 55 asymptomatic diabetic patients and similarly concluded that DM was independently associated with decreased reservoir and conduit strain function, impaired autonomic function as measured by reduced heart rate variability, and a compensatory increased booster pump function (94). Such remodeling influences of DM on LA add to the risk of development of AF, an already feared outcome of diabetic cardiomyopathy (95). Table 1 summarizes the studies evaluating LA strain mechanics in various disease conditions.

\section{Current limitations in clinical applications of LA strain mechanics}

Widespread utility of LA strain mechanics for assessment of disease severity and prognostication is limited at present. This is partly contributed to by a relative lack of robust data on the correlation between different LA parameters with outcomes, as well as accurate non-invasive modalities for LA volume estimation. The assessment of LA function in a comprehensive manner requires a combined assessment of both LA pressure and LA volume. In the absence of modalities for direct assessment of LA pressure, estimates are indirectly measured, and can be influenced by both LV dysfunction and primary abnormalities in the LA, making it difficult to differentiate between the various contributing factors. Additionally, rhythm disturbances are not accounted for in LA strain assessment. Additionally, the heterogeneity in the LA strain values obtained from different software platforms should be noted.

\section{Future perspectives and conclusions}

Traditionally, imaging parameters relating to LV function and structure have been typically used to assist with risk stratification and help guide decisions for interventions. More recently, the assessment of LA function and structure has gained increasing popularity for early detection of disease severity, and the scope has gradually

Table 1 A review of existing literature on the clinical utility of LA strain mechanics

\begin{tabular}{|c|c|c|c|}
\hline $\begin{array}{l}\text { Clinical } \\
\text { application }\end{array}$ & Author, publication year & $\begin{array}{l}\text { Study sample } \\
\text { size }\end{array}$ & Outcome \\
\hline \multirow[t]{3}{*}{ AF } & Cameli et al., 2017 (29) & 79 & $\begin{array}{c}\text { Global LASr }<8.1 \% \text {, predicted LAA thrombus and reduced LAA emptying } \\
\text { velocity }\end{array}$ \\
\hline & Lacalzada-Almeida et al., 2019 (30) & 98 & SRa decrease related to evolution of new onset AF or stroke or both \\
\hline & Yoon et al., 2015 (31) & 52 & LAS $\leq 30.9 \%$ was associated with AF progression \\
\hline \multirow[t]{3}{*}{ HTN } & Mondillo et al., 2011 (34) & 121 & Decreases in all LAS and SR indices, exception of peak LASRa \\
\hline & Hennawy et al., 2018 (35) & 50 & Global LASr lower in the HTN despite normal LAVI \\
\hline & Karakurt et al., 2019 (36) & 29 & Peak LAS and SR lower in HTN with preserved LAEF and LVEF \\
\hline HFrEF & Carluccio et al., 2018 (42) & 405 & LASr independent prognostic marker \\
\hline
\end{tabular}

Table 1 (continued) 
Table 1 (continued)

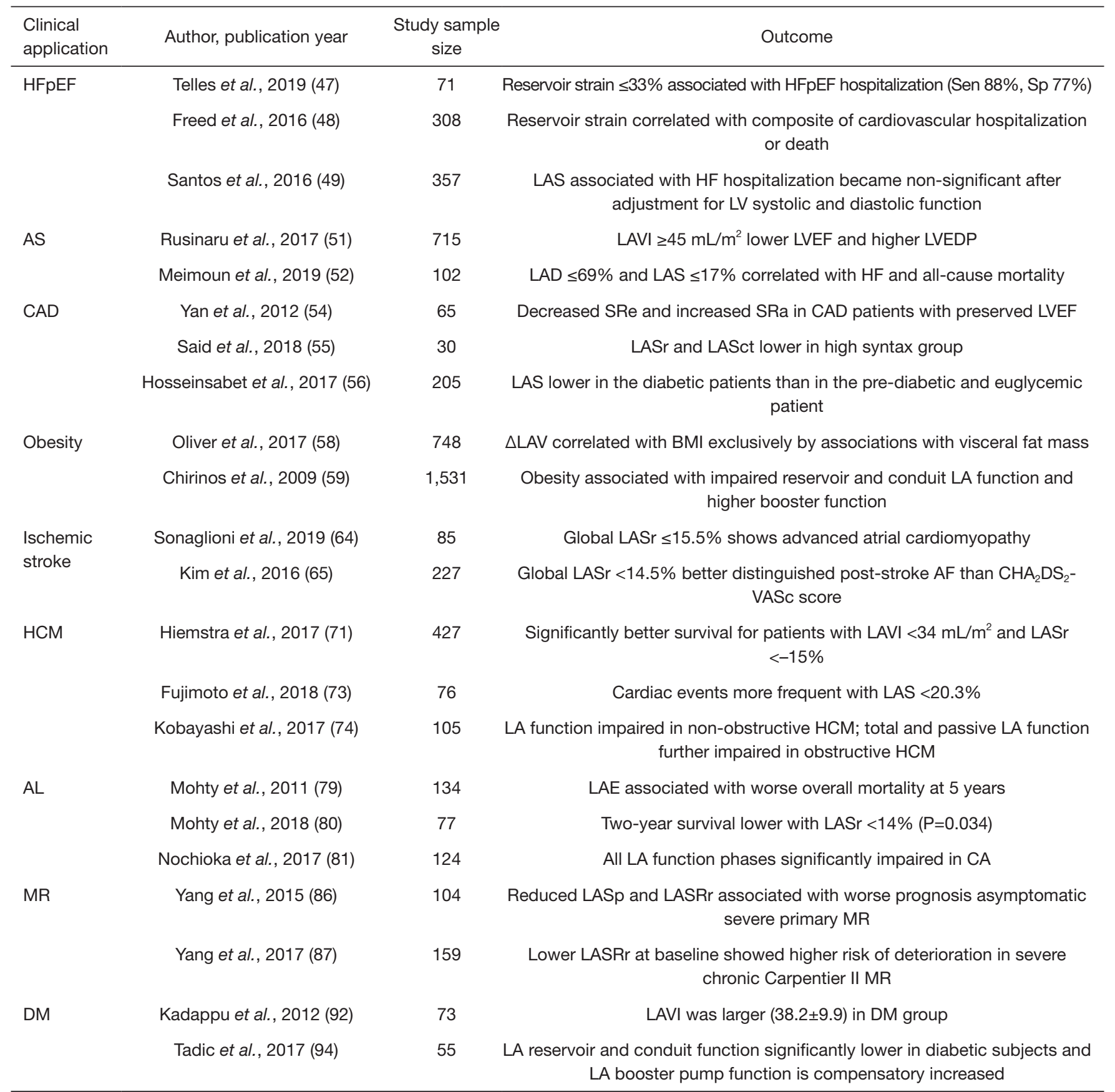

LA, left atrial; HF, heart failure; AF, atrial fibrillation; LASr, LA reservoir strain; LAA, LA appendage; SRa, strain rate during late filling; LAS, LA strain; SR, strain rate; HTN, hypertension; LAVI, LA volume index; LAEF, LA ejection fraction; LVEF, left ventricular ejection fraction; HFrEF, HF with reduced ejection fraction; LVEDP, left ventricular end diastolic pressure; HFpEF, HF with preserved ejection fraction; Sen, sensitivity; Sp, specificity; AS, aortic stenosis; LAD, left anterior descending; CAD, coronary artery disease; SRe, strain rate during early filling; LAV, LA volume; BMI, body mass index; HCM, hypertrophic cardiomyopathy; AL, amyloidosis; LAE, LA enlargement; CA, cardiac amyloidosis; MR, mitral regurgitation; LASp, peak LA strain; LASRr, strain rate in LA filling phase; DM, diabetes mellitus. 
expanded to include assessment of valvular pathologies, cardiomyopathies, and systemic diseases, such as HTN and DM. With the advent of newer technologies for strain measurement, the understanding of LA function through the different stages of the cardiac cycle is evolving. Furthermore, the introduction of standardized indices has laid the foundation for future larger studies, and potential future inclusion of LA strain mechanics in consensus guidelines with the emergence of more robust prospective data. The current data regarding the clinical utility of LA strain mechanics are largely derived from small studies. This highlights the need for further large prospective multicenter studies, investigating the clinical applications of LA strain mechanics in various cardiovascular conditions.

\section{Acknowledgments}

Funding: None.

\section{Footnote}

Provenance and Peer Review: This article was commissioned by the editorial office, Cardiovascular Diagnosis and Therapy for the series "Heart Valve Disease". The article has undergone external peer review.

Reporting Checklist: The authors have completed the NARRATIVE REVIEW reporting checklist. Available at http://dx.doi.org/10.21037/cdt-20-461

Conflicts of Interest: All authors have completed the ICMJE uniform disclosure form (available at http://dx.doi. org/10.21037/cdt-20-461). The series "Heart Valve Disease" was commissioned by the editorial office without any funding or sponsorship. BX served as the unpaid Guest Editor of the series. Dr. AK reports personal fees from Swedish Orphan Biovitrum AB (Sobi). The authors have no other conflicts of interest to declare.

Ethical Statement: The authors are accountable for all aspects of the work in ensuring that questions related to the accuracy or integrity of any part of the work are appropriately investigated and resolved.

Open Access Statement: This is an Open Access article distributed in accordance with the Creative Commons Attribution-NonCommercial-NoDerivs 4.0 International License (CC BY-NC-ND 4.0), which permits the non- commercial replication and distribution of the article with the strict proviso that no changes or edits are made and the original work is properly cited (including links to both the formal publication through the relevant DOI and the license). See: https://creativecommons.org/licenses/by-nc-nd/4.0/.

\section{References}

1. Abhayaratna WP, Seward JB, Appleton CP, et al. Left atrial size: physiologic determinants and clinical applications. J Am Coll Cardiol 2006;47:2357-63.

2. Salvo GD, Pergola V, Fadel B, et al. Strain echocardiography and myocardial mechanics: from basics to clinical applications. J Cardiovasc Echogr 2015;25:1-8.

3. Miller DM. Evidence that interfacial transport is ratelimiting during passive cell membrane permeation. Biochim Biophys Acta 1991;1065:75-81.

4. Pagel PS, Kehl F, Gare M, et al. Mechanical function of the left atrium: new insights based on analysis of pressure-volume relations and Doppler echocardiography. Anesthesiology 2003;98:975-94.

5. Mitchell JH, Shapiro W. Atrial function and the hemodynamic consequences of atrial fibrillation in man. Am J Cardiol 1969;23:556-67.

6. Prioli A, Marino P, Lanzoni L, et al. Increasing degrees of left ventricular filling impairment modulate left atrial function in humans. Am J Cardiol 1998;82:756-61.

7. Lemire F, Tajik AJ, Hagler DJ. Asymmetric left atrial enlargement; an echocardiographic observation. Chest 1976;69:779-81.

8. Sahn DJ, DeMaria A, Kisslo J, et al. Recommendations regarding quantitation in M-mode echocardiography: results of a survey of echocardiographic measurements. Circulation 1978;58:1072-83.

9. Kircher B, Abbott JA, Pau S, et al. Left atrial volume determination by biplane two-dimensional echocardiography: validation by cine computed tomography. Am Heart J 1991;121:864-71.

10. Rodevan O, Bjornerheim R, Ljosland M, et al. Left atrial volumes assessed by three- and two-dimensional echocardiography compared to MRI estimates. Int J Card Imaging 1999;15:397-410.

11. Poutanen T, Jokinen E, Sairanen H, et al. Left atrial and left ventricular function in healthy children and young adults assessed by three dimensional echocardiography. Heart 2003;89:544-9.

12. Appleton CP, Galloway JM, Gonzalez MS, et al. Estimation of left ventricular filling pressures using two- 
dimensional and Doppler echocardiography in adult patients with cardiac disease. Additional value of analyzing left atrial size, left atrial ejection fraction and the difference in duration of pulmonary venous and mitral flow velocity at atrial contraction. J Am Coll Cardiol 1993;22:1972-82.

13. Appleton CP, Gonzalez MS, Basnight MA. Relationship of left atrial pressure and pulmonary venous flow velocities: importance of baseline mitral and pulmonary venous flow velocity patterns studied in lightly sedated dogs. J Am Soc Echocardiogr 1994;7:264-75.

14. Manning WJ, Silverman DI, Katz SE, et al. Atrial ejection force: a noninvasive assessment of atrial systolic function. J Am Coll Cardiol 1993;22:221-5.

15. Manning WJ, Silverman DI, Katz SE, et al. Impaired left atrial mechanical function after cardioversion: relation to the duration of atrial fibrillation. J Am Coll Cardiol 1994;23:1535-40.

16. Thomas L, Hoy M, Byth K, et al. The left atrial function index: a rhythm independent marker of atrial function. Eur J Echocardiogr 2008;9:356-62.

17. Sardana M, Lessard D, Tsao CW, et al. Association of left atrial function index with atrial fibrillation and cardiovascular disease: the Framingham Offspring Study. J Am Heart Assoc 2018;7:e008435.

18. Rosca M, Lancellotti P, Popescu BA, et al. Left atrial function: pathophysiology, echocardiographic assessment, and clinical applications. Heart 2011;97:1982-9.

19. Urheim S, Edvardsen T, Torp H, et al. Myocardial strain by Doppler echocardiography. Validation of a new method to quantify regional myocardial function. Circulation 2000;102:1158-64.

20. Mor-Avi V, Lang RM, Badano LP, et al. Current and evolving echocardiographic techniques for the quantitative evaluation of cardiac mechanics: ASE/EAE consensus statement on methodology and indications endorsed by the Japanese Society of Echocardiography. J Am Soc Echocardiogr 2011;24:277-313.

21. Wang Y, Li Z, Fei H, et al. Left atrial strain reproducibility using vendor-dependent and vendor-independent software. Cardiovasc Ultrasound 2019;17:9.

22. Badano LP, Kolias TJ, Muraru D, et al. Standardization of left atrial, right ventricular, and right atrial deformation imaging using two-dimensional speckle tracking echocardiography: a consensus document of the EACVI/ASE/Industry Task Force to standardize deformation imaging. Eur Heart J Cardiovasc Imaging 2018;19:591-600.

23. Pathan F, D'Elia N, Nolan MT, et al. Normal ranges of left atrial strain by speckle-tracking echocardiography: a systematic review and meta-analysis. J Am Soc Echocardiogr 2017;30:59-70.e8.

24. Safir-Mardanloo A, Khorsand Askari M, Lotfi Tokaldany $M$, et al. Normal values for tissue velocity and strain rate imaging parameters of left and right atrial myocardium in normal subjects. Echocardiography 2019;36:521-7.

25. Morris DA, Takeuchi M, Krisper M, et al. Normal values and clinical relevance of left atrial myocardial function analysed by speckle-tracking echocardiography: multicentre study. Eur Heart J Cardiovasc Imaging 2015;16:364-72.

26. Parikh MG, Aziz Z, Krishnan K, Madias C, et al. Usefulness of transesophageal echocardiography to confirm clinical utility of CHA2DS2-VASc and CHADS2 scores in atrial flutter. Am J Cardiol 2012;109:550-5.

27. Tani T, Tanabe K, Ono M, et al. Left atrial volume and the risk of paroxysmal atrial fibrillation in patients with hypertrophic cardiomyopathy. J Am Soc Echocardiogr 2004;17:644-8.

28. Vaziri SM, Larson MG, Benjamin EJ, et al. Echocardiographic predictors of nonrheumatic atrial fibrillation. The Framingham Heart Study. Circulation 1994;89:724-30.

29. Cameli M, Lunghetti S, Mandoli GE, et al. Left atrial strain predicts pro-thrombotic state in patients with nonvalvular atrial fibrillation. J Atr Fibrillation 2017;10:1641.

30. Lacalzada-Almeida J, Izquierdo-Gomez MM, GarciaNiebla J, et al. Advanced interatrial block is a surrogate for left atrial strain reduction which predicts atrial fibrillation and stroke. Ann Noninvasive Electrocardiol 2019;24:e12632.

31. Yoon YE, Oh IY, Kim SA, et al. Echocardiographic predictors of progression to persistent or permanent atrial fibrillation in patients with paroxysmal atrial fibrillation (E6P study). J Am Soc Echocardiogr 2015;28:709-17.

32. Hwang HJ, Choi EY, Rhee SJ, et al. Left atrial strain as predictor of successful outcomes in catheter ablation for atrial fibrillation: a two-dimensional myocardial imaging study. J Interv Card Electrophysiol 2009;26:127-32.

33. Tumuklu MM, Erkorkmaz U, Ocal A. The impact of hypertension and hypertension-related left ventricle hypertrophy on right ventricle function. Echocardiography 2007;24:374-84.

34. Mondillo S, Cameli M, Caputo ML, et al. Early detection of left atrial strain abnormalities by speckle-tracking in hypertensive and diabetic patients with normal left atrial size. J Am Soc Echocardiogr 2011;24:898-908. 
35. Hennawy B, El Kilany W, Galal H, et al. Role of speckle tracking echocardiography in detecting early left atrial dysfunction in hypertensive patients. Egypt Heart J 2018;70:217-23.

36. Karakurt A, Yildiz C, Yildiz A, et al. Early detection strain/ strain rate and time to strain/strain rate abnormalities for left atrial mechanical function in hypertensive patients. Acta Cardiol 2019;74:141-51.

37. Degirmenci H, Duman H, Demirelli S, et al. Assessment of effect of irbesartan and nebivolol on the left atrium volume and deformation in the patients with mildmoderate hypertension. Eur Rev Med Pharmacol Sci 2014;18:781-9.

38. Sharifov OF, Gupta H. What is the evidence that the tissue Doppler index E/e' reflects left ventricular filling pressure changes after exercise or pharmacological intervention for evaluating diastolic function? A systematic review. J Am Heart Assoc 2017;6:e04766.

39. Cameli M, Lisi M, Mondillo S, et al. Left atrial longitudinal strain by speckle tracking echocardiography correlates well with left ventricular filling pressures in patients with heart failure. Cardiovasc Ultrasound 2010;8:14.

40. Cameli M, Mandoli GE, Loiacono F, et al. Left atrial strain: a new parameter for assessment of left ventricular filling pressure. Heart Fail Rev 2016;21:65-76.

41. Cameli M, Sparla S, Losito M, et al. Correlation of left atrial strain and doppler measurements with invasive measurement of left ventricular end-diastolic pressure in patients stratified for different values of ejection fraction. Echocardiography 2016;33:398-405.

42. Carluccio E, Biagioli P, Mengoni A, et al. Left atrial reservoir function and outcome in heart failure with reduced ejection fraction. Circ Cardiovasc Imaging 2018;11:e007696.

43. Kurt M, Tanboga IH, Aksakal E, et al. Relation of left ventricular end-diastolic pressure and $\mathrm{N}$-terminal pro-brain natriuretic peptide level with left atrial deformation parameters. Eur Heart J Cardiovasc Imaging 2012;13:524-30.

44. Malagoli A, Rossi L, Bursi F, et al. Left atrial function predicts cardiovascular events in patients with chronic heart failure with reduced ejection fraction. J Am Soc Echocardiogr 2019;32:248-56.

45. Dernellis JM, Stefanadis CI, Zacharoulis AA, et al. Left atrial mechanical adaptation to long-standing hemodynamic loads based on pressure-volume relations. Am J Cardiol 1998;81:1138-43.
46. Wan SH, Vogel MW, Chen HH. Pre-clinical diastolic dysfunction. J Am Coll Cardiol 2014;63:407-16.

47. Telles F, Nanayakkara S, Evans S, et al. Impaired left atrial strain predicts abnormal exercise haemodynamics in heart failure with preserved ejection fraction. Eur J Heart Fail 2019;21:495-505.

48. Freed BH, Daruwalla V, Cheng JY, et al. Prognostic utility and clinical significance of cardiac mechanics in heart failure with preserved ejection fraction: importance of left atrial strain. Circ Cardiovasc Imaging 2016. doi: 10.1161/ CIRCIMAGING.115.003754.

49. Santos AB, Roca GQ, Claggett B, et al. Prognostic relevance of left atrial dysfunction in heart failure with preserved ejection fraction. Circ Heart Fail 2016;9:e002763.

50. Rossi A, Tomaino M, Golia G, et al. Usefulness of left atrial size in predicting postoperative symptomatic improvement in patients with aortic stenosis. Am J Cardiol 2000;86:567-70, A9-10.

51. Rusinaru D, Bohbot Y, Salaun E, et al. Determinants of left atrial volume index in patients with aortic stenosis: a multicentre pilot study. Arch Cardiovasc Dis 2017;110:525-33.

52. Meimoun P, Djebali M, Botoro T, et al. Left atrial strain and distensibility in relation to left ventricular dysfunction and prognosis in aortic stenosis. Echocardiography 2019;36:469-77.

53. Aguero J, Galan-Arriola C, Fernandez-Jimenez R, et al. Atrial infarction and ischemic mitral regurgitation contribute to post-MI remodeling of the left atrium. J Am Coll Cardiol 2017;70:2878-89.

54. Yan P, Sun B, Shi H, et al. Left atrial and right atrial deformation in patients with coronary artery disease: a velocity vector imaging-based study. PLoS One 2012;7:e51204.

55. Said KM, Nassar AI, Fouad A, et al. Left atrial deformation analysis as a predictor of severity of coronary artery disease. Egypt Heart J 2018;70:353-9.

56. Hosseinsabet A, Mohseni-Badalabadi R, Jalali A. Two-dimensional speckle-tracking echocardiography evaluation of left atrial function according to glycemic state in patients with coronary artery disease. Cardiovasc Endocrinol 2017;6:101-8.

57. Ayer JG, Sholler GF, Celermajer DS. Left atrial size increases with body mass index in children. Int J Cardiol 2010;141:61-7.

58. Oliver W, Matthews G, Ayers CR, et al. Factors associated with left atrial remodeling in the general population. Circ 
Cardiovasc Imaging 2017;10:e005047.

59. Chirinos JA, Rietzschel ER, De Buyzere ML, et al. Arterial load and ventricular-arterial coupling: physiologic relations with body size and effect of obesity. Hypertension 2009;54:558-66.

60. Mahabadi AA, Lehmann N, Kalsch H, et al. Association of epicardial adipose tissue and left atrial size on non-contrast CT with atrial fibrillation: the Heinz Nixdorf Recall Study. Eur Heart J Cardiovasc Imaging 2014;15:863-9.

61. Chirinos JA, Sardana M, Satija V, et al. Effect of Obesity on Left Atrial Strain in Persons Aged 35-55 Years (The Asklepios Study). Am J Cardiol 2019;123:854-61.

62. D'Souza M, Carlson N, Fosbol E, et al. CHA2DS2VASc score and risk of thromboembolism and bleeding in patients with atrial fibrillation and recent cancer. Eur J Prev Cardiol 2018;25:651-8.

63. Arora L, Singh VN, Gupta P, et al. Band gap engineered $\mathrm{P} 3 \mathrm{HT} / \mathrm{CdPbS}$ composites for utilization of low energy photons. J Nanosci Nanotechnol 2014;14:4995-5001.

64. Sonaglioni A, Vincenti A, Baravelli M, et al. Prognostic value of global left atrial peak strain in patients with acute ischemic stroke and no evidence of atrial fibrillation. Int J Cardiovasc Imaging 2019;35:603-13.

65. Kim D, Shim CY, Cho IJ, et al. Incremental value of left atrial global longitudinal strain for prediction of post stroke atrial fibrillation in patients with acute ischemic stroke. J Cardiovasc Ultrasound 2016;24:20-7.

66. Kramer CM, Reichek N, Ferrari VA, et al. Regional heterogeneity of function in hypertrophic cardiomyopathy. Circulation 1994;90:186-94.

67. Yang H, Woo A, Monakier D, et al. Enlarged left atrial volume in hypertrophic cardiomyopathy: a marker for disease severity. J Am Soc Echocardiogr 2005;18:1074-82.

68. Maron BJ, Roberts WC, Epstein SE. Sudden death in hypertrophic cardiomyopathy: a profile of 78 patients. Circulation 1982;65:1388-94.

69. Mizukoshi K, Suzuki K, Yoneyama K, et al. Early diastolic function during exertion influences exercise intolerance in patients with hypertrophic cardiomyopathy. J Echocardiogr 2013;11:9-17.

70. Siontis KC, Geske JB, Ong K, et al. Atrial fibrillation in hypertrophic cardiomyopathy: prevalence, clinical correlations, and mortality in a large high-risk population. J Am Heart Assoc 2014;3:e001002.

71. Hiemstra YL, Debonnaire P, Bootsma M, et al. Global longitudinal strain and left atrial volume index provide incremental prognostic value in patients with hypertrophic cardiomyopathy. Circ Cardiovasc Imaging 2017;10:e05706.

72. Sanada H, Shimizu M, Sugihara N, et al. Increased left atrial chamber stiffness in hypertrophic cardiomyopathy. Br Heart J 1993;69:31-5.

73. Fujimoto K, Inoue K, Saito $M$, et al. Incremental value of left atrial active function measured by speckle tracking echocardiography in patients with hypertrophic cardiomyopathy. Echocardiography 2018;35:1138-48.

74. Kobayashi Y, Wheeler M, Finocchiaro G, et al. Left atrial function and phenotypes in asymmetric hypertrophic cardiomyopathy. Echocardiography 2017;34:843-50.

75. Nemes A, Kormanyos A, Domsik P, et al. Normal reference values of three-dimensional speckle-tracking echocardiography-derived left atrial strain parameters (results from the MAGYAR-Healthy Study). Int J Cardiovasc Imaging 2019;35:991-8.

76. Guan J, Mishra S, Falk RH, et al. Current perspectives on cardiac amyloidosis. Am J Physiol Heart Circ Physiol 2012;302:H544-52.

77. Falk RH. Diagnosis and management of the cardiac amyloidoses. Circulation 2005;112:2047-60.

78. Klein AL, Hatle LK, Taliercio CP, et al. Prognostic significance of Doppler measures of diastolic function in cardiac amyloidosis. A Doppler echocardiography study. Circulation 1991;83:808-16.

79. Mohty D, Pibarot P, Dumesnil JG, et al. Left atrial size is an independent predictor of overall survival in patients with primary systemic amyloidosis. Arch Cardiovasc Dis 2011;104:611-8.

80. Mohty D, Petitalot V, Magne J, et al. Left atrial function in patients with light chain amyloidosis: a transthoracic 3D speckle tracking imaging study. J Cardiol 2018;71:419-27.

81. Nochioka K, Quarta CC, Claggett B, et al. Left atrial structure and function in cardiac amyloidosis. Eur Heart J Cardiovasc Imaging 2017;18:1128-37.

82. Linhart A, Kampmann C, Zamorano JL, et al. Cardiac manifestations of Anderson-Fabry disease: results from the international Fabry outcome survey. Eur Heart J 2007;28:1228-35.

83. Biegstraaten M, Arngrimsson R, Barbey F, et al. Recommendations for initiation and cessation of enzyme replacement therapy in patients with Fabry disease: the European Fabry Working Group consensus document. Orphanet J Rare Dis 2015;10:36.

84. Pichette M, Serri K, Page M, et al. Impaired left atrial function in Fabry disease: a longitudinal speckletracking echocardiography study. J Am Soc Echocardiogr 2017;30:170-9.e2. 
85. Nishimura RA, Otto CM, Bonow RO, et al. 2017 AHA/ ACC focused update of the 2014 AHA/ACC guideline for the management of patients with valvular heart disease: a report of the American College of Cardiology/American Heart Association Task Force on Clinical Practice Guidelines. J Am Coll Cardiol 2017;70:252-89.

86. Yang LT, Liu YW, Shih JY, et al. Predictive value of left atrial deformation on prognosis in severe primary mitral regurgitation. J Am Soc Echocardiogr 2015;28:1309-17.e4.

87. Yang LT, Tsai WC, Luo CY, et al. Role of left atrial reservoir strain rate in left atrial remodeling in severe mitral regurgitation. J Med Ultrasound 2017;25:16-23.

88. Ring L, Rana BS, Wells FC, et al. Atrial function as a guide to timing of intervention in mitral valve prolapse with mitral regurgitation. JACC Cardiovasc Imaging 2014;7:225-32.

89. Shaw JE, Sicree RA, Zimmet PZ. Global estimates of the prevalence of diabetes for 2010 and 2030. Diabetes Res Clin Pract 2010;87:4-14.

90. Nichols GA, Hillier TA, Erbey JR, et al. Congestive heart failure in type 2 diabetes: prevalence, incidence, and risk factors. Diabetes Care 2001;24:1614-9.

91. Ernande L, Rietzschel ER, Bergerot C, et al. Impaired myocardial radial function in asymptomatic patients with type 2 diabetes mellitus: a speckle-tracking imaging study. J Am Soc Echocardiogr 2010;23:1266-72.

92. Kadappu KK, Boyd A, Eshoo S, et al. Changes in left atrial volume in diabetes mellitus: more than diastolic dysfunction? Eur Heart J Cardiovasc Imaging 2012;13:1016-23.

93. Muranaka A, Yuda S, Tsuchihashi K, et al. Quantitative assessment of left ventricular and left atrial functions by strain rate imaging in diabetic patients with and without hypertension. Echocardiography 2009;26:262-71.

94. Tadic M, Vukomanovic V, Cuspidi C, et al. Left atrial phasic function and heart rate variability in asymptomatic diabetic patients. Acta Diabetol 2017;54:301-8.

95. Tadic M, Cuspidi C. Type 2 diabetes mellitus and atrial fibrillation: from mechanisms to clinical practice. Arch Cardiovasc Dis 2015;108:269-76.
Cite this article as: Jain V, Ghosh R, Gupta M, Saijo Y, Bansal A, Farwati M, Marcus R, Klein A, Xu B. Contemporary narrative review on left atrial strain mechanics in echocardiography: cardiomyopathy, valvular heart disease and beyond. Cardiovasc Diagn Ther 2021;11(3):924-938. doi: $10.21037 /$ cdt-20-461 